\title{
Exploring black hole superkicks
}

\author{
Bernd Brügmann, ${ }^{1}$ José A. González, ${ }^{1,2}$ Mark Hannam, ${ }^{1}$ Sascha Husa, ${ }^{1}$ and Ulrich Sperhake ${ }^{1}$ \\ ${ }^{1}$ Theoretical Physics Institute, University of Jena, 07743 Jena, Germany \\ ${ }^{2}$ Instituto de Física y Matemáticas, Universidad Michoacana de San Nicolás de Hidalgo, \\ Edificio C-3, Cd. Universitaria. C. P. 58040 Morelia, Michoacán, México \\ (Received 7 August 2007; published 27 June 2008)
}

\begin{abstract}
Recent calculations of the recoil velocity in black-hole binary mergers have found kick velocities of $\approx 2500 \mathrm{~km} / \mathrm{s}$ for equal-mass binaries with antialigned initial spins in the orbital plane. In general the dynamics of spinning black holes can be extremely complicated and are difficult to analyze and understand. In contrast, the "superkick" configuration is an example with a high degree of symmetry that also exhibits exciting physics. We exploit the simplicity of this test case to study more closely the role of spin in black-hole recoil and find that the recoil is with good accuracy proportional to the difference between the $(l=2, m= \pm 2)$ modes of $\Psi_{4}$, the major contribution to the recoil occurs within $30 M$ before and after the merger, and that this is after the time at which a standard post-Newtonian treatment breaks down. We also discuss consequences of the $(l=2, m= \pm 2)$ asymmetry in the gravitational wave signal for the angular dependence of the signal-to-noise ratio and the mismatch of the gravitational wave signals corresponding to the north and south poles.
\end{abstract}

DOI: 10.1103/PhysRevD.77.124047

PACS numbers: 04.25.dg, 04.30.Db, 95.30.Sf, 98.80.Jk

\section{INTRODUCTION}

More than 40 years after Hahn and Lindquist started the numerical investigation of colliding black holes [1], a series of breakthroughs starting in 2005 [2-4] has turned the quest for stable black hole inspiral simulations into a gold rush.

A particular focus of the last few months has been the socalled recoil or rocket effect due to "beamed" emission of gravitational radiation [5-7]. By momentum conservation, radiation of energy in a preferred direction corresponds to a loss of linear momentum and the black hole that results from the merger thus recoils from the center-of-mass frame with speeds of up to a few thousand $\mathrm{km} / \mathrm{s}$. The velocity of this "kick" depends on the configuration of the system (e.g., the mass ratio and spins) and details of the merger dynamics, but not on the total mass (velocity is dimensionless in geometric units). From an astrophysical point of view, the recoil effect is particularly interesting for massive black holes with masses $>10^{5} M_{\odot}$, which exist at the center of most galaxies and may have a substantial impact on the structure and formation of their host galaxies. Observational consequences of large recoil have recently been considered in $[8,9]$.

The largest recoil effects have so far been found [10,11] for a particularly simple configuration suggested in [12] based on [13]: equal-mass binaries with (initially) antialigned spins in the orbital plane. Based on numerical simulations for different configurations and a postNewtonian approximation [13], an estimate of $1300 \mathrm{~km} / \mathrm{s}$ had been obtained for this configuration with maximal spin [14]. The kicks found in full numerical simulations are however even larger, e.g. $2500 \mathrm{~km} / \mathrm{s} \mathrm{[10]} \mathrm{or} 1800 \mathrm{~km} / \mathrm{s}$ $[11,12]$ for nonmaximally spinning black holes. This is of the order of $1 \%$ of the speed of light, and can be larger than the escape velocity of about $2000 \mathrm{~km} / \mathrm{s}$ from giant elliptical galaxies. Extrapolating current numerical results for nonmaximal spins to maximally spinning black holes predicts recoil velocities of up to $\approx 4000 \mathrm{~km} / \mathrm{s}$ [11]. Smaller but still significant kick velocities have been found for several different types of black hole configurations [15-22]. Estimations of the probabilities to obtain different kick velocities for different mass ratios and high spins were studied in [23].

The parameter space of the inspiral of spinning black holes is very large, and although its full exploration will require numerical methods, analytical understanding and approximations will be crucial to render the task economical. The purpose of the present paper is to obtain a better understanding of the physics that leads to the large kick results recently observed, and, in particular, to compare with post-Newtonian approximations, and see where such approximations are accurate, and where they (currently) break down.

We will refer to a configuration similar to that described in $[10,11]$, i.e., two equal-mass black holes with spins antialigned and in the orbital plane, as a superkick configuration. The superkick configuration exhibits " $\pi$ symmetry," i.e. it is invariant under a rotation by an angle $\pi$ about an axis perpendicular to the initial orbital plane. It follows from this symmetry that linear momentum will not be radiated in the $x$ or $y$ directions, but only in the $z$ direction. As a consequence, the center of mass will remain at $(x=0, y=0)$ but can move in the $z$ direction.

The paper is organized as follows. In Sec. II we briefly summarize our numerical methods and list the simulations we have performed. Section III analyzes several aspects of the dynamics of the "superkick" configurations, in par- 
ticular, the comparison with post-Newtonian dynamics and various aspects of the $(l=2, m= \pm 2)$ asymmetry. Consequences of this asymmetry for the angular dependence of the signal-to-noise ratio (SNR) and the mismatch of the gravitational wave signals, exemplified by the extreme case of the north and south poles, are discussed in Sec. IV. The paper concludes with a discussion section and four appendices that contain post-Newtonian equations we use in this paper, as well as a number of small results concerning the dynamics of moving-puncture simulations. Specifically, we describe our procedure for estimating quasicircular inspiral parameters for spinning binaries, summarize some aspects of the post-Newtonian (PN) treatment of spinning binaries, show that the coordinate speeds of the punctures can be easily related to their momenta by a standard PN expression (and that, in particular, one does not need to understand the complicated properties of the spacetime near the punctures to explain the puncture's coordinate speeds), and, finally, we compare results in the Arnowitt-Deser-Misner transverse traceless and harmonic gauges.

\section{NUMERICAL METHODS AND SUMMARY OF SIMULATIONS}

In this section we will summarize our numerical methods for evolving black hole binary spacetimes (largely by directing the reader to the relevant references) and specify the numerical simulations we performed. The various simulations will be motivated more fully later in the paper; for now we give an overview for later reference.

We performed numerical simulations with the BAM [24,25] and LEAN [26] codes, with modifications discussed in [10]. Both codes start with black hole binary puncture initial data $[27,28]$ generated using a pseudospectral code [29], and they are evolved with the $\chi$ variant of the moving-puncture [3,4] version of the BaumgarteShapiro-Shibata-Nakamura [30,31] formulation of the $3+$ 1 Einstein evolution equations [32]. The gravitational waves emitted by the binary are calculated from the Newman-Penrose scalar $\Psi_{4}$, and the details of this procedure for BAM and LEAN are given in [24,26], respectively.

The parameters of our simulations are summarized in Table I. Each black hole has mass $M_{i}$ (with mass parameter $m_{i}$ in the puncture data construction [28]), and the total mass is $M=M_{1}+M_{2}$. The black holes have a coordinate separation of $D$. In all runs the punctures are placed on the $y$ axis at $y= \pm D / 2$ and given momenta $p_{x}$ and spins $S=$ $0.723 M_{i}^{2}=0.2$. The spins are aligned with the $y$ direction, except for the runs in the " $\alpha$-series," which are characterized by $S_{y}= \pm S \cos \alpha$ and $S_{x}=\mp S \sin \alpha$.

The $\alpha$ series and $P$ series simulations used modifications of the MI configuration described in [10]. This configuration was chosen because the results showed clean fourthorder convergence and high accuracy. We have found that the resolution requirements increase significantly for simulations of spinning black holes, and the MI configuration, with a small initial separation and therefore short evolution time, provided a convenient starting point for our study; these simulations also capture most of the important dynamics that we wish to study.

The $\alpha$ and $P$ series simulations were performed with the grid setup $\chi_{\eta=2}[6 \times 44: 4 \times 88: 6][88: 5.82]$ in the notation of [24], i.e., the six inner boxes had $44^{3}$ points, the four outer boxes had $88^{3}$ points, the resolution on the finest level is $M / 88$, and the resolution at the outer boundary is $5.82 M$. Convergence tests were performed for the $\alpha=0$ case (which is the same as the MI configuration in [10]) with inner-box sizes of 40, 44, 48, and corresponding resolutions. Clean fourth-order convergence of the linear momentum radiation flux $d P_{z} / d t$ is shown in Fig. 1. Also shown is convergence in the puncture separation, which is not expected to last beyond the merger time of about $t=$ $88 \mathrm{M}$ since the separation between the two punctures inside the common apparent horizon quickly approaches zero [24].

Further simulations were performed using either larger initial separation and/or quasicircular orbit parameters (calculated according the prescription given in Appendix A). These are indicated D6 (for $D=6 M$ ) and D8 (for $D=8.2 M$ ) in the table. The D8 simulation was performed using the LEAN code, while all others were performed with BAM. The grid setup for the D6 simulations was the same as for the $\alpha$ and $P$ series, and the convergence test referred to later used inner-box sizes of 44,48 , and 52 points. The D8 simulation used a grid setup $\chi_{\eta=1}[2 \times$ $133: 1 \times 155: 2 \times 133: 3 \times 67: 9]\left[44: \frac{32}{11}\right]$, where the innermost three levels with 67 points are centered around either hole and follow the motion of the puncture.

Experimentally we have observed that the resolutions used in the $\alpha$ and $P$ series simulations are not sufficient to

TABLE I. Physical parameters of the simulations performed for this paper.

\begin{tabular}{|c|c|c|c|c|c|c|c|c|}
\hline Simulation & $D$ & $m_{i}$ & $p_{x}$ & $M$ & $\alpha$ & Est. $t_{\text {merger }}$ & Orbits & $V_{\text {kick }}(\mathrm{km} / \mathrm{s})$ \\
\hline$\alpha$ series & 6.514 & 0.363 & 0.133 & 1.052 & $\begin{array}{c}0 \leq \alpha<2 \pi \\
\delta \alpha=\pi / 6\end{array}$ & $80-85 M$ & 1.0 & $-2700 \leq V_{\text {kick }} \leq 2700$ \\
\hline$P$ series & 6.514 & 0.363 & $\begin{array}{c}0.13034 \leq p_{x} \leq 0.13566 \\
\delta p=0.003325\end{array}$ & 1.052 & 0 & $75-90 M$ & $0.75-1.25$ & $2286 \leq V_{\text {kick }} \leq 2690$ \\
\hline D6 & 6.0 & 0.296 & 0.1382 & 1.0 & 0 & $123 M$ & 1.75 & 2100 \\
\hline D8 & 8.198 & 0.2875 & 0.11 & 1.0 & 0 & $260 M$ & 2.5 & 725 \\
\hline
\end{tabular}



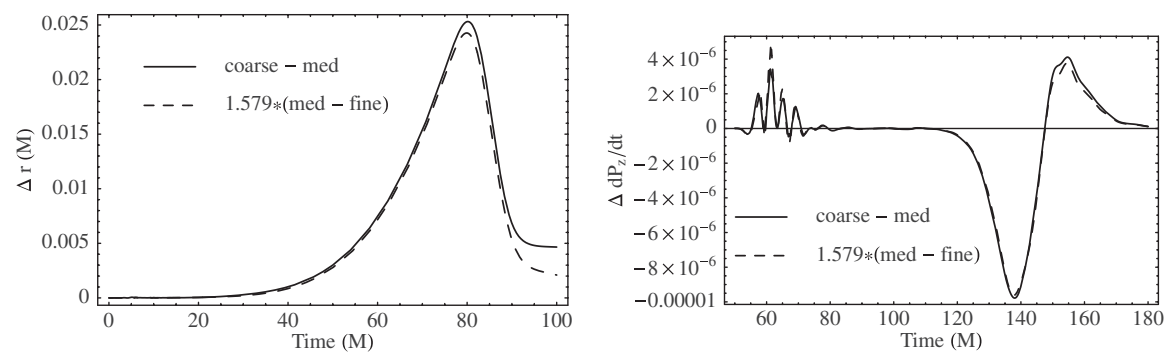

FIG. 1. Convergence plots for the puncture separation $r$ and the linear momentum radiation flux in the $z$ direction, $d P_{z} / d t$ obtained for model $\alpha=0$ of the $\alpha$ series. The plots are scaled consistent with fourth-order convergence. After merger at about $t=88 M$ convergence in the puncture separation is lost (as expected).

obtain clean convergence for evolutions of spinning black holes orbiting for longer periods of time. It thus appears that the good convergence results for these particular series are largely due to the close initial separation of the black holes, which results in a rather low merger time of about $88 \mathrm{M}$. When the black holes are placed further apart (or even making the seemingly innocuous change of choosing quasicircular orbit initial parameters for the same separation as the $\alpha$ series simulations), convergence is lost before the black holes merge. We expect that fourth-order convergence would be obtained if sufficiently high resolutions were used, but the extra computational expense was not necessary for the analysis in this paper.

In the D6 simulations we find that the puncture separation and linear momentum radiation flux $d P_{z} / d t$ converge

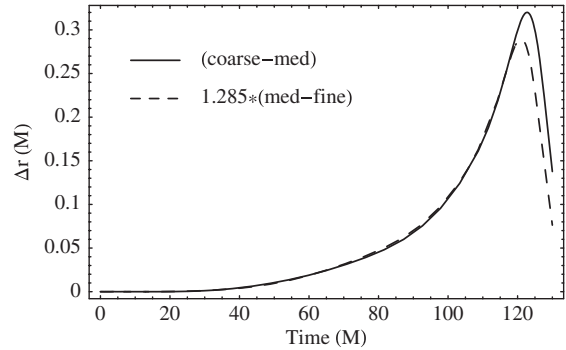

well for up to $15 \mathrm{M}$ before merger, as shown in Figs. 2 and 3. Note that since the waves are extracted at $R_{\mathrm{ex}}=50 \mathrm{M}$, we need to take into account a time lag of roughly $50 \mathrm{M}$ when comparing times related to puncture motion and wave extraction. These simulations will be used only for discussions of the qualitative behavior, and for analysis at early times, when we are confident that the results are reliable. Similarly the long D8 LEAN simulation will be used only for qualitative comparison with post-Newtonian results.

\section{ANALYSIS OF SUPERKICK DYNAMICS}

We begin by describing the dynamics of two black holes in a superkick configuration with varying degrees of sim-

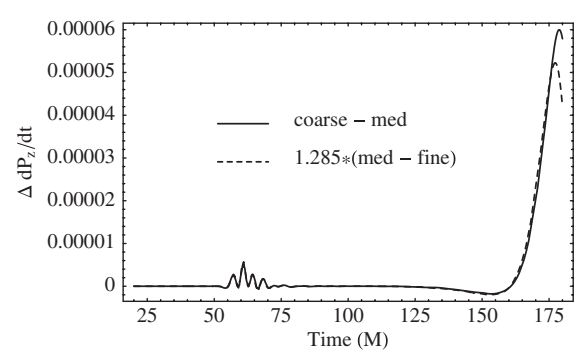

FIG. 2. Convergence of the puncture separation and $d P_{z} / d t$ as functions of time for evolutions of model D6. Results are scaled for fourth-order convergence. We see that fourth-order convergence is lost in the puncture separation at about $t=115 M$, which corresponds to roughly $t=165 M$ in quantities from waves extracted at $R_{\mathrm{ex}}=50 \mathrm{M}$, which is about when we see a loss of convergence in $d P_{z} / d t$. Note that we cut the plot at $t \approx 175 M$ when convergence is lost.
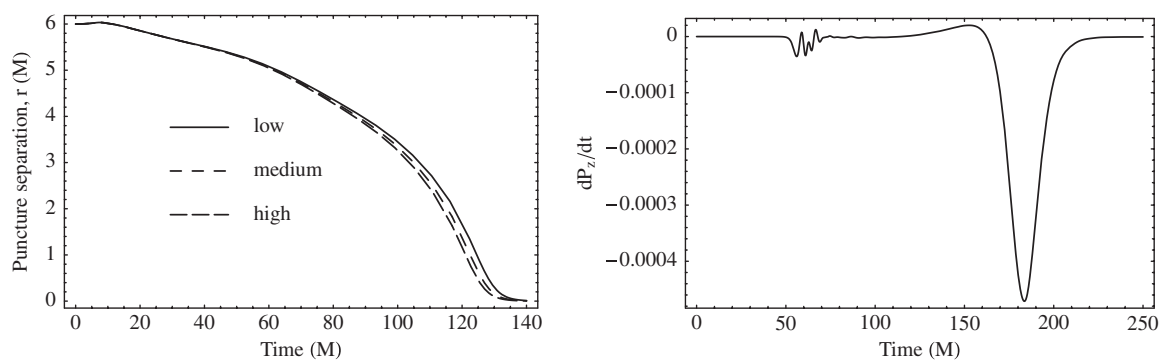

FIG. 3. Puncture separation and $d P_{z} / d t$ as functions of time for the evolutions of model D6. Results from low, medium, and high resolution simulations are shown. Only the highest resolution is shown for $d P_{z} / d t$. 
plicity, in order to build up a clearer picture of the physics and to motivate the simulations and analysis we have performed. In the simplest picture (Sec. III B) the spin decouples from the orbital dynamics; a more complex picture includes spin precession effects (Sec. IIIC), and considering the dynamics in full general relativity (GR) in Sec. IIID allows us to study the merger regime, from which most of the kick effect originates. The full GR results can then be directly compared with PN predictions, which we do in Sec. IIIE. We discuss the spin of the final black hole in Sec. III F.

\section{A. Kick velocity and $l=2, m= \pm 2$ symmetry breaking}

As noted before, the superkick configuration exhibits " $\pi$ symmetry" ( $\phi \rightarrow \phi+\pi)$, thus linear momentum will not be radiated in the $x$ or $y$ directions, but radiation of linear momentum in the $z$ direction is allowed, and the center of mass will move only in the $z$ direction.

As in nonspinning equal-mass binary simulations, almost all of the energy is radiated in the $l=2, m= \pm 2$ modes: the maximal relative deviation of the energy in those modes from the total energies is roughly $2 \%$, neglecting the contribution from the junk radiation. This fact, and the symmetry discussed in the preceding paragraph, leads us to expect that we should be able to directly relate the kick in the $z$ direction to the imbalance between the $m=2$ and $m=-2$ modes, i.e., the difference in energy that is radiated toward the "north" and "south" hemispheres. Using the special relativistic relation $|\vec{p}|=E$ between the momentum and energy of a wave packet (traveling at the speed of light), we expect a relation

$$
p_{z}=f \times\left(E_{22}-E_{2-2}\right)
$$

for the radiated momentum in the $z$ direction, where $E_{22}$ and $E_{2-2}$ are the energies radiated in the $l=2, m= \pm 2$ modes, and $f$ is a geometric factor. Here $0 \leq f<1$ expresses the fact that the radiation is smeared out in solid angle rather than sharply peaked in the direction of the poles. Neglecting all modes but $l=2, m= \pm 2$, we assume a wave signal in the form $\Psi_{4}=\kappa F(t) Y_{22}^{-2}(\theta, \phi)+$ $\lambda \bar{F}(t) Y_{2-2}^{-2}(\theta, \phi)$, where $\kappa, \lambda$ are real numbers $(\kappa=\lambda$ in the nonspinning equal-mass case), $F(t)$ is a complex timedependent function, and the $Y_{2 \pm 2}^{-2}$ are the spin-weighted spherical harmonics

$$
\begin{aligned}
& Y_{2-2}^{-2}=\sqrt{\frac{5}{64 \pi}}(1-\cos \theta)^{2} e^{-2 i \phi}, \\
& Y_{22}^{-2}=\sqrt{\frac{5}{64 \pi}}(1+\cos \theta)^{2} e^{2 i \phi} .
\end{aligned}
$$

Inserting this ansatz into the expressions for radiated energy and linear momentum (see e.g. Eqs. (48) and (49) in [24]) we obtain

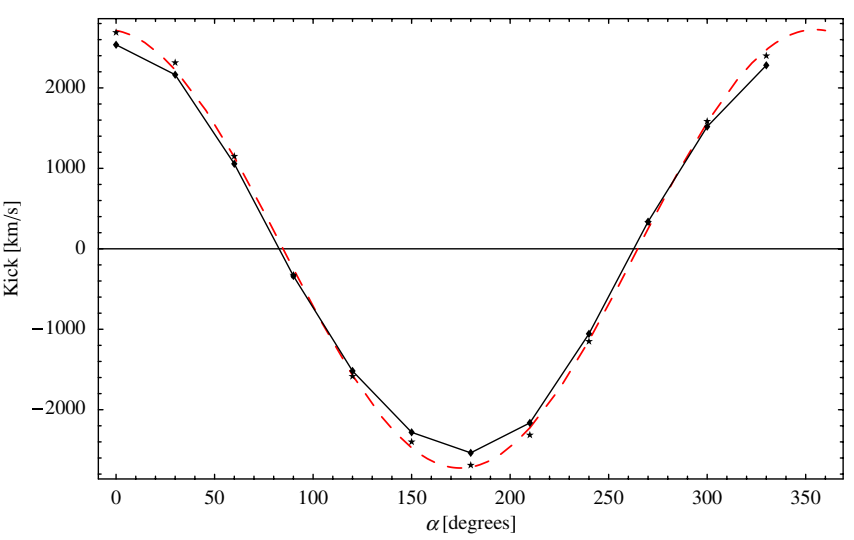

FIG. 4 (color online). Comparison of the kick velocity in $\mathrm{km} / \mathrm{s}$ according to Eq. (1), for a range of angles $\alpha$. Data points for the measured kick and the estimate Eq. (1) are shown, the points corresponding to the energy differences are connected. An analytical fit, $v_{z}=2725 \cos (0.98 \pi+\alpha) \mathrm{km} / \mathrm{s}$, to the measured kick is shown as a dashed line. Note that Eq. (1) slightly underestimates the kick, which is consistent since it neglects contributions from higher-order multipoles $l>2$.

$$
\begin{gathered}
\frac{d E}{d t}=\frac{r^{2}}{16 \pi}\left(\kappa^{2}+\lambda^{2}\right)\left|\int_{\infty}^{t} F(\tilde{t}) d \tilde{t}\right|^{2}, \\
\frac{d P_{z}}{d t}=\frac{2}{3} \frac{r^{2}}{16 \pi}\left(\kappa^{2}-\lambda^{2}\right)\left|\int_{\infty}^{t} F(\tilde{t}) d \tilde{t}\right|^{2} .
\end{gathered}
$$

Consequently the value of the geometric factor $f$ can be determined as $f=2 / 3$. We find this relationship satisfied to very good accuracy in our numerical evolutions, as shown in Fig. 4.

The relative asymmetry in the energies emitted in the $l=2, m= \pm 2$ modes, $2 E_{22} /\left(E_{22}+E_{2-2}\right)$, (this quantity is unity when there is no symmetry breaking) is plotted in Fig. 5, showing a maximal excess of roughly $40 \%$. An analytic fit for extraction radius $R_{\text {ext }}=50 M$ is

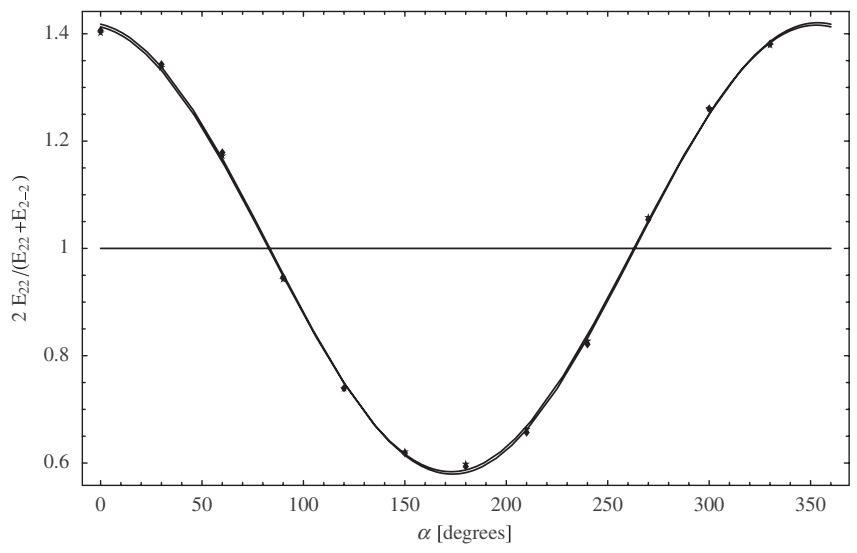

FIG. 5. Excess energy in the $l=2, \quad m=2$ mode, $2 E_{22} /\left(E_{22}+E_{2-2}\right)$ plotted for extraction radii $R_{\text {ext }}=30 M$, and $50 \mathrm{M}$. The curves are the analytical fits for both extraction radii, see Eq. (5). Clearly, there is no significant dependence of this ratio on extraction radius. 


$$
\frac{2 E_{22}}{\left(E_{22}+E_{2-2}\right)}=1+0.416 \cos (0.125+\alpha) \text {. }
$$

In the extreme case this fit corresponds to $E_{22} / E_{2-2} \approx 2.4$. For this fit the $95 \%$ confidence level statistical error is roughly $20 \%$ for the phase, and roughly $2 \%$ for the amplitude of the oscillation. Fits corresponding to the extraction radii $R_{\text {ext }}=30 M$ and $75 M$ give consistent results with Eq. (5) within the statistical error bars.

\section{B. Simplest assumption: Spin decouples from black hole dynamics}

As a first approximation of the dynamics, we may imagine that the black holes behave like (force-free) gyroscopes in flat spacetime, and as they orbit each other their individual spin vectors do not change direction. For example, if the spins were originally $\mathbf{S}_{1}=(0, S, 0)$ and $\mathbf{S}_{2}=$ $(0,-S, 0)$, these vectors would be constant throughout the evolution. If we also assume that the spins do not noticeably influence the motion in the $x-y$ plane, then simulations that start with the same initial separation and momenta will display the same dynamics no matter how the spins are directed in the orbital plane. This is the situation at the firstPN approximation, since spin-orbit and spin-spin couplings enter at higher order.

This picture is surprisingly close to the observed dynamics in numerical simulations. Figure 6 shows the orbital motion in the first six simulations of the $\alpha$ series, each differing only in the initial directions of the spins [i.e., $\mathbf{S}_{1,2}=S(\mp \sin \alpha, \pm \cos \alpha, 0)$ and $\alpha=0 \ldots \pi$ in steps of $\pi / 6$ ]. The motion shows differences as $\alpha$ is varied (shown in the lower panels of the figure), but these differences are
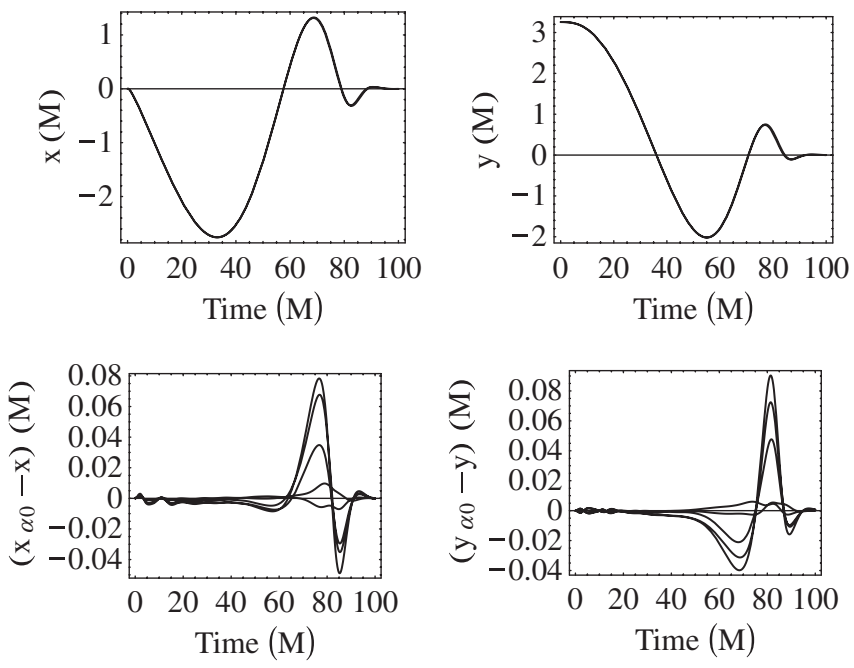

FIG. 6. The $x$ and $y$ motion of one of the punctures in six simulations from the $\alpha$ series. The dynamics in the $x$-y plane are almost identical for all of the simulations (only one curve is actually visible in the upper panels). The small variations in the motion (measured with respect to the $\alpha=0$ simulation) are shown in the lower panels. very small. In contrast, the resulting kick from these simulations, shown in Fig. 4, displays a clear sinusoidal dependence on the angle-the kick varies from $\approx-2500 \mathrm{~km} / \mathrm{s}$ to $+2500 \mathrm{~km} / \mathrm{s}$. (Note that, since these values were calculated at a small radiation extraction radius $R_{\mathrm{ex}}=50 M$, the values in the plot are systematically higher than the correct values by about $10 \%$ ). Similar figures were also shown in [11].

We might conclude from these results that the final kick depends only on the initial spin magnitude and direction of each black hole. One may write an expression similar to Eq. (1) in [11], which for the superkick case reduces to

$$
V_{K}=k \cos \left(\alpha-\alpha_{0}\right)
$$

and determine the constants $k$ and $\alpha_{0}$, which are approximately given by $k \approx 2500 \mathrm{~km} / \mathrm{s}$ and $\alpha_{0} \approx 0$ for our data. If this simple picture was correct, and the spins really did behave as gyroscopes in flat spacetime, then (6) would allow us to determine $\alpha_{0}=0$ as the spin direction that produces the maximum kick.

\section{The effect of spin precession during inspiral}

Needless to say, the real situation is more complicated. The picture of the black holes' spins as gyroscopes in flat spacetime is valid only at 1PN order; at higher postNewtonian orders and in full GR the spin directions evolve during the inspiral. We expect that the magnitude of the kick depends on the magnitude and direction of the spins when the black holes are close to merger. The spin configuration at merger time is a function of the initial configuration plus precession effects during the evolution.

The precession effects can be seen in Fig. 7, which shows $\left(S_{x}, S_{y}, S_{z}\right)$ as a function of time for the $\alpha=0$ simulation described earlier. Here the spins were calculated from the black holes' apparent horizons, using the coordinate-based method outlined in [33] and also used in [21]. We see that the $x$ and $y$ components of the spin show noticeable precession during the last orbit, and after $80 \mathrm{M}$ of evolution the spin vector has rotated by $\pi / 2$. The $z$-component shows small oscillations around zero, but these are not well resolved with the current accuracy of the code. Note, however, by comparison with Fig. 6 that the period of the oscillation is roughly half an orbital period, which is consistent with the post-Newtonian equations in Appendix B. We will further comment on the comparison of the spin precession with PN results in Sec. III E. After the formation of a common apparent horizon, at about $t \approx$ $88 M$, the $S_{x}$ and $S_{y}$ quickly drop to zero, and $S_{z}$ jumps to its final value, corresponding to the spin of the final black hole, $S_{z} / M_{f}^{2} \approx 0.7$, where $M_{f}$ is the mass of the final black hole. We also compute the spin of the final black hole from quasinormal ringdown in Sec. III F as $S_{z} / M_{f}^{2} \approx 0.69$. Note that this value of the final spin is also the value for nonspinning equal-mass inspirals, see e.g. [24]. 

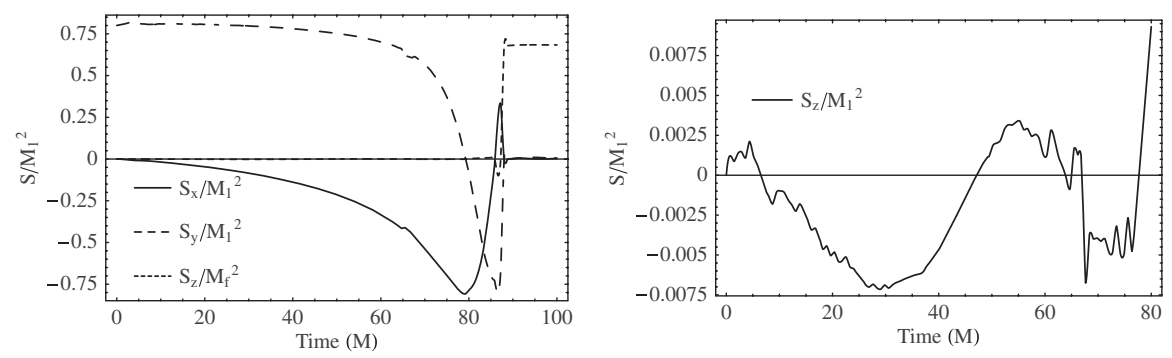

FIG. 7. Evolution of the spins $S_{x}, S_{y}$, and $S_{z}$ of one of the black holes over the course of the $\alpha=0$ simulation. The $x$ and $y$ components of the spin show noticeable precession during the last orbit; the spin has rotated by $\pi / 2$ after $80 M$ of evolution. By contrast, the $z$ component (right-hand panel) displays small oscillations around zero; these oscillations are not well resolved at the current accuracy of the code. Merger occurs at $t \approx 88 M$, at which time $S_{x}$ and $S_{y}$ drop to zero. The spin $S_{z} / M_{f}^{2}$ jumps to a final value of 0.723 corresponding to the merged black hole, reflecting the conversion of orbital angular momentum into the spin of the final object.

As an example of the dependence of the final kick on parameters besides $\alpha$, Fig. 8 shows the final kick for $\alpha=0$ simulations with differing values of the initial momenta of the black holes (the $P$-series in Table I). Changing the initial momenta causes the merger time to change, and this means that the spins have more or less time to evolve, and are therefore in different directions when the black holes merge. The initial spin directions are, however, the same for all of these simulations. Varying the initial momenta in this way also alters the ellipticity of the system. However, the variations we make to the momenta are small (less than 5\% between the smallest and largest values) and at this initial separation do not have a large effect on the eccentricity. It is unlikely that these small changes in the eccentricity are the cause of the large variation in the final kick (around $400 \mathrm{~km} / \mathrm{s}$ ), and they would certainly not account for the turning point in Fig. 8; the true cause is different spin alignment near or at merger.

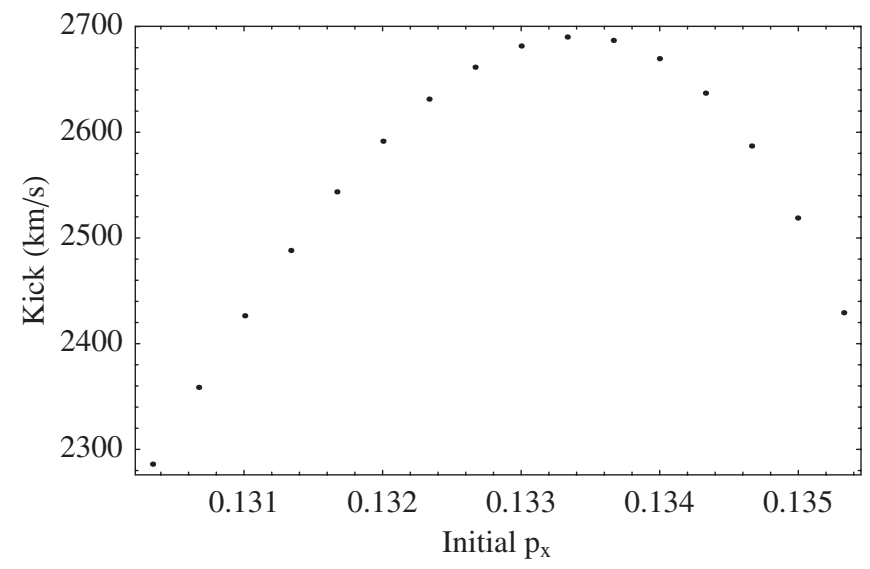

FIG. 8. The final kick as a function of the initial momenta of the black holes for simulations with the initial spin angle $\alpha=0$. The differences between the smallest and largest merger time is only about $15 \mathrm{M}$; these differences allow a little more or less spin precession, and therefore have a strong effect on the final recoil, which varies between 2300 and $2700 \mathrm{~km} / \mathrm{s}$.
This leads us back to Eq. (1) in [11], which was originally written in terms of the spin angles at merger. Above we formulated Eq. (6) in terms of the initial angle, but it would hold equally well if, instead of choosing $\alpha=\alpha(t=$ $0)$ we were to choose some fixed $t_{0}$ and use $\alpha=\alpha\left(t=t_{0}\right)$ in Eq. (6). Only the phase constant $\alpha_{0}$ would change. The results shown in Fig. 8 suggest that we will also see oscillatory behavior if we make a plot of the final kick versus merger time for a series of runs with the same initial spin directions.

In conclusion, given some superkick initial configuration we need to know both the spin angle $\alpha$ and the time until merger in order to predict the magnitude of the final kick from initial data.

\section{Duration of the recoil}

Simplified models aside, we know that the final kick is due to an integration of $d P_{z} / d t$ over the entire evolution, and $d P_{z} / d t$ will be a complicated function of the instantaneous spin directions. A post-Newtonian version of this function is given in [13] and in Appendix B. Figure 9 shows $d P_{z} / d t$ as a function of time for three simulations

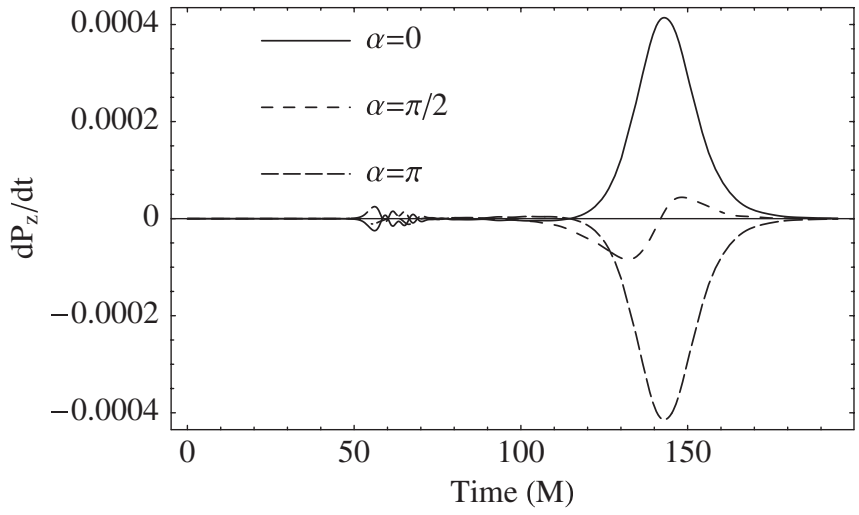

FIG. 9. Plot of $d P_{z} / d t$ as a function of time for the $\alpha=0$, $\pi / 2, \pi$ simulations. Most of the linear momentum is radiated over a $60 \mathrm{M}$ period of time, centered roughly around the merger time. 
in the $\alpha$ series. We make several observations about these plots. The main contribution to the final kick originates from a time period of about $60 \mathrm{M}$. Before that time the contribution is negligible, which is even clearer in Fig. 3, which shows $d P_{z} / d t$ for the quasicircular model D6 which has a longer inspiral phase. We also see in Fig. 9 that the function $d P_{z} / d t$ obtained for simulations with final kick of 2500,0 , and $-2500 \mathrm{~km} / \mathrm{s}$ does not only differ by a mere rescaling. Instead, the curve obtained for $\alpha=\pi / 2$ exhibits an additional oscillation.

We would like to relate $d P_{z} / d t$ to the motion of the punctures, but this is not trivial. The radiation is extracted at some radius $R_{\mathrm{ex}}$, and plotting this as a function of retarded time $r-R_{\mathrm{ex}}$ gives only a crude estimate of what is happening in the vicinity of the black holes at any given time. One could try to improve this estimate by using instead the luminosity distance (see for example [34]), but we choose to simply look at the puncture motion directly. We can calculate the coordinate acceleration of the punctures in the $z$ direction, $a_{z}(t)=d^{2} z(t) / d t^{2}$. Figure 10 shows the acceleration of one of the punctures in the $\alpha=0, \pi / 2, \pi$ simulations. A vertical line indicates the time at which a common apparent horizon forms, and thus gives us an indication of how much of the motion is due to effects before merger, and how much after merger.

Although most of the final kick is generated before merger, Fig. 10 suggests that $d P_{z} / d t$ is not negligible after the merger. Referring back to the plot of $d P_{z} / d t$ in Fig. 9, the waves from the merger can be estimated to reach the radiation extraction sphere between $138 M$ (the time when the common apparent horizon forms, $88 M$, plus the ex-

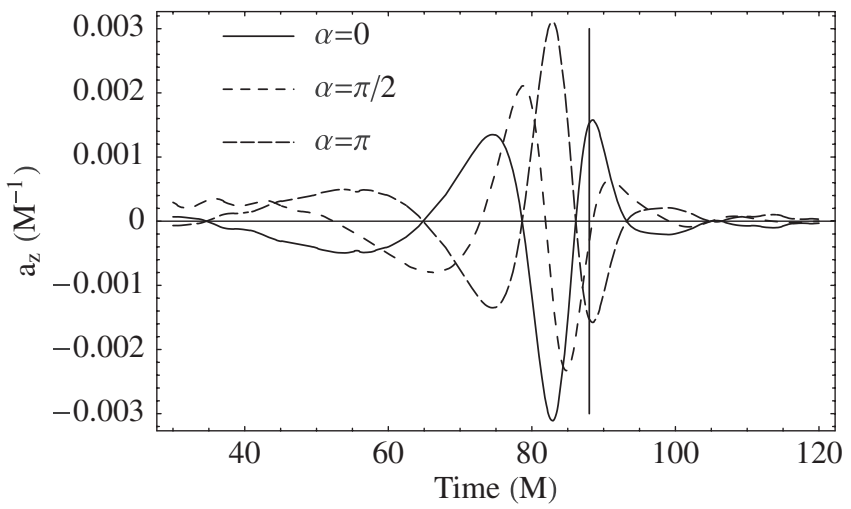

FIG. 10. The coordinate acceleration of one of the punctures in the $z$ direction for the $\alpha=0, \pi / 2, \pi$ simulations. The vertical line represents the time of first formation of a common apparent horizon. The curves notably differ from those in Fig. 3, in particular, they show significant extra oscillations, while they have roughly the same amplitude. The plot essentially confirms that the gauge dependence of the puncture motion in this direction is not well understood (in contrast with the orbital motion discussed in Appendix C), which prevents us from drawing quantitative conclusions about the kick velocity from the coordinate acceleration. traction radius, 50M) and $155 M$ (when the final black hole's ringdown can clearly be said to have begun, by observing that the wave amplitude has a clean exponential decay). Whatever time within this range we choose to denote as "merger," it is clear that a significant contribution to the final kick arises after that time.

\section{E. Comparison with post-Newtonian predictions}

In Fig. 10 we see that there is a significant contribution to the kick after the black holes merge. Before the merger, the main contribution comes from the $30 \mathrm{M}$ before merger. During this time the black holes are at a separation of $D<$ $3.5 \mathrm{M}$, and at this separation it is questionable whether a post-Newtonian description of the radiated linear momentum is meaningful. We will now make a comparison between our numerical results and the predictions from the 2.5PN-accurate expressions in [13].

Appendices B, C, and D summarize the techniques we use to compare post-Newtonian and numerical results. Briefly, Appendix B lists the expressions for the spin evolution and radiated linear momenta as found in [13]. These expressions were derived in the harmonic gauge. Our initial data are instead in the ADMTT gauge (up to 2PN accuracy [35]), and although it is not obvious how well we remain in the ADMTT gauge during evolution (but see [36] for a result that shows excellent agreement for larger separations), we would like to see how much the results differ between the two gauges. Appendix D thus gives the expressions necessary to transform the numerical quantities, which are assumed to be in the ADMTT gauge, to the harmonic gauge. To do this we need to calculate the momenta of the punctures as they evolve. Appendix C gives 2PN expressions that relate the puncture's speeds (again assumed to be in the ADMTT gauge) to momenta as given in Eq. (C2). We see in Appendix D that in fact the ADMTT $\rightarrow$ harmonic transformation makes little difference to our results over the time when the PN approximation is valid. This result may not be surprising, but it quantifies any confusion that may arise when we compare results in the two gauges and eliminates any major concern that our results may change drastically if we were to perform our simulations in the harmonic gauge.

As an aside, these formulas explain the speed at which the punctures move in a black hole binary movingpuncture simulation; the puncture speeds and momenta are not related by the Newtonian formula $p=m v$, but instead to good accuracy by its $2 \mathrm{PN}$ counterpart.

Before considering the radiated linear momentum, we compare the post-Newtonian predictions for the spin evolution with our numerical results. For this purpose we use simulation D8, which has a merger time of around 260M. Figure 11 shows the evolution of $S_{x}, S_{y}$, and $S_{z}$ for simulation D8, compared with the predictions from Eqs. (B1). We see that there is very good qualitative agreement in $S_{x}$ and $S_{y}$, even close to merger, which occurs at around 

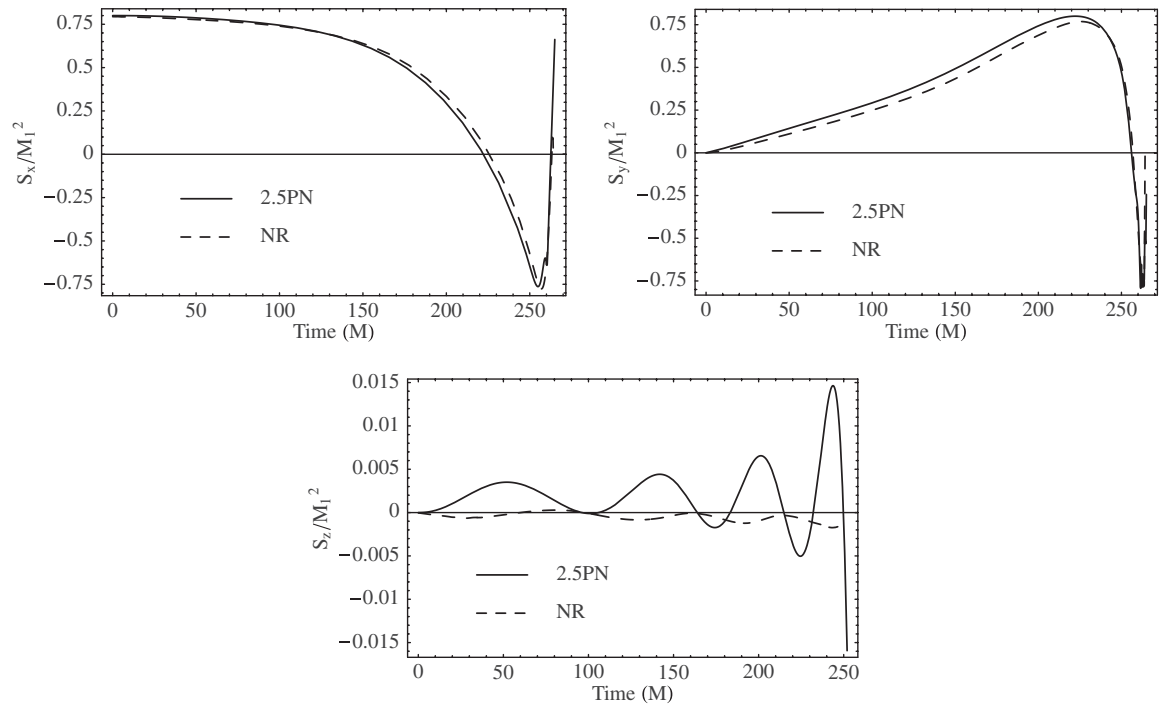

FIG. 11. One black hole's spin as a function of time for simulation D8. Also shown is the 2.5PN prediction for the spin evolution, with the puncture dynamics $\left\{x_{i}, v_{i}\right\}$ used in the spin evolution Eqs. (B1). The agreement is very good for $S_{x}$ and $S_{y}$, but poor for $S_{z}$. This may once again be due to the numerical motion in the $z$ direction being far more gauge dependent than the motion in the $x$ - $y$ plane. At late times $S_{x}$ and $S_{y}$ vanish, whereas $S_{z}$ is found to correspond to the angular momentum of the final black hole, compare Fig. 7 and Sec. III F.

$t=260 M$. The $z$ component does not agree at all well with the $2.5 \mathrm{PN}$ prediction, but we again note that the puncture motion in the $z$ direction may be much more gauge dependent than the motion in the $x-y$ plane (the PN equations are given in the center-of-mass frame, while the puncture motion certainly is not), and it is the positions and speeds of the punctures that we use when evaluating the right-hand sides of Eqs. (B1). Furthermore, it is not clear how well the numerical determination of the spin based on apparent horizons works in this context. Note also that the absolute error is very small. The frequency of the oscillations of the numerical simulation is rather close to the $\mathrm{PN}$ result, which is approximately twice the orbital frequency when precession effects are small (cf. Appendix B). After merger, a few $M$ after the end of the figure, $S_{z}$ jumps to its final value of around 0.7, and $S_{x}$ and $S_{y}$ drop to zero.

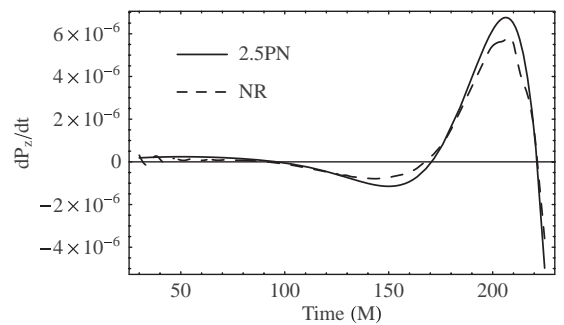

In the case of the radiated linear momentum flux $d P_{z} / d t$ and the final kick, we know that the assumptions underlying the post-Newtonian expressions break down when the black holes are very close. Equations (B3) diverge as $1 / r^{5}$ as the particles' separation $r \rightarrow 0$, so it is clear that a sensible estimate of the kick cannot be made by simply integrating this equation. What has been done in the past (see for example [37]) is to assume a cutoff separation and integrate the post-Newtonian expression up to that point. We will now show that this approach is unlikely to give correct results in the superkick case.

Figure 12 shows the function $d P_{z} / d t$ compared to the numerical values for the D8 simulation at a retarded time $t-54.5 \mathrm{M}$, chosen to line up the early oscillations in $d P_{z} / d t$, and close to a naive guess of the retarded time for the extraction radius $R_{\mathrm{ex}}=50 M$. The post-Newtonian val-

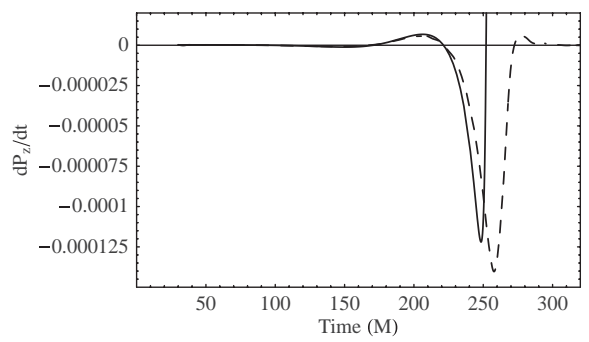

FIG. 12. Comparison of the numerical $d P_{z} / d t$ with that predicted by Eqs. (B3). The left-hand plot shows early times, up to $t=$ $225 \mathrm{M}$, while the right-hand plot includes the merger. A time shift of $54.5 \mathrm{M}$ was applied to the value calculated from the numerical wave extraction, to approximately take into account the wave travel time between the punctures and the wave extraction sphere by lining up the peak at $t \approx 200 \mathrm{M}$. The numerical relativity and $2.5 \mathrm{PN}$ results agree qualitatively at early times, but diverge quickly near merger, presumably due to the $1 / r^{5}$ term in Eq. (B3). 
ues of $d P_{z} / d t$ were calculated as follows. Equations (B3) require as input the positions, speeds, and spins of the two black holes. Rather than integrate the full post-Newtonian equations of motion, we simply enter the appropriate quantities from a numerical evolution. This allows us to compare, moment by moment, the post-Newtonian and numerical predictions of $d P_{z} / d t$ for two particles (or black holes) with the $\left\{x_{i}, p_{i}, S_{i}\right\}$ configuration.

In Fig. 12 we once again see good qualitative agreement at early times (left-hand plot). At late times (right-hand plot) the post-Newtonian prediction diverges, due to the $1 / r^{5}$ term in Eqs. (B3). What is most striking about this plot is that the disagreement between numerical and postNewtonian results becomes serious around $50 M$ before merger, which is just before the time when the major contribution to the recoil begins in Figs. 3, 9, and 10. This suggests that, at least in the special case of superkick configurations, if we integrate the post-Newtonian $d P_{z} / d t$ up to the point where its accuracy breaks down, we will grossly underestimate the value of the final kick.

In order to accurately estimate the value of the kick analytically, one would need to make a much more sophisticated choice of cutoff separation, and then perhaps match to a close-limit analysis. Such a procedure was applied in $[38,39]$ to nonspinning binaries, and may well be applicable in the spinning case. One may also be able to get good results from a more careful post-Newtonian analysis, as was performed (also in the nonspinning case) in [40,41]. We would expect that the superkick case would be an extreme and particularly interesting test of such methods.

\section{F. Spin of the final black hole from ringdown}

In Sec. III C we found that the spin of the final black hole as read off from the black hole horizon is $J_{z} / M^{2} \approx 0.7$. Here we will also determine the dimensionless Kerr spin parameter $a=J_{z} / M^{2}$ from the quasinormal ringdown gravitational wave signal of the slowest decaying spinweighted spheroidal harmonic mode $[42,43]$, which we measure by projecting it onto the $l=2, m= \pm 2$ spinweighted spherical harmonics. These projected $l=2$, $m= \pm 2$ waveforms are split into amplitude and phase according to $\psi_{4}=A(t) \exp (i \varphi(t))$; we then perform analytical fits to the waveform for $170 \leq t / M \leq 230$, where we see both a clean exponential decrease of the wave amplitude and a linear increase of the gravitational wave phase (corresponding to a constant frequency). Performing independent fits with a linear function for the wave phase and an exponential for the amplitude we obtain values for the complex ringdown frequency $\omega_{\mathrm{QNM}}$. In order to factor out the overall mass scale we then perform a lookup of the dimensionless quantity $\operatorname{Im}\left(\omega_{\mathrm{QNM}}\right) / \operatorname{Re}\left(\omega_{\mathrm{QNM}}\right)$ (i.e. essentially the inverse quality factor) in a table of quasinormal mode frequencies [44].

We will consider, in particular, data from the $\alpha$ series, see Table I. For the extraction radii $R_{\mathrm{ex}}=30 M, 50 M, 75 M$

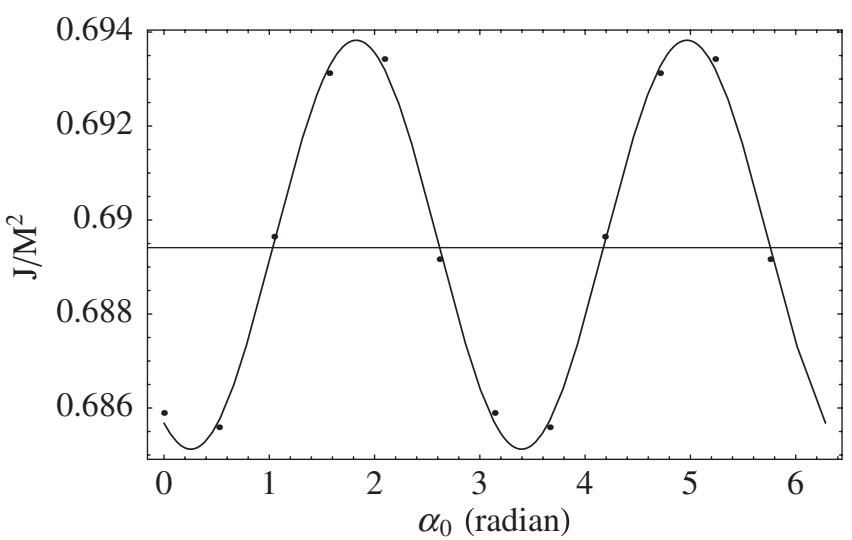

FIG. 13. The plot shows numerical results for $J / M^{2}=\frac{a_{22}+a_{2-2}}{2}$ (points), obtained for extraction radius $R_{\mathrm{ex}}=50 \mathrm{M}$ for the final black hole and an analytical fit (solid curve) - in the text we conclude that the final Kerr spin parameter does not show significant variations (which might be further reduced by increased accuracy of the wave extraction).

both the $l=2, m= \pm 2$ results can very well be fit with an analytic expression of the form $a_{0}+a_{1} \cos \left(\alpha+\varphi_{1}\right)+$ $a_{2} \cos \left(2 \alpha+\varphi_{2}\right)$, see Fig. 13. At each extraction radius we get consistent results for the amplitudes $a_{0}, a_{2}$ and the phase shifts $\varphi_{1}, \varphi_{2}$ for the $m=-2,2$ modes, but we get the opposite sign for $a_{1}$ for the $m=-2$ and $m=2$ modes. For $a_{0}$ we get $(0.6963,0.6891,0.6891) \pm 5 \times 10^{-4}$ (statistical error) for extraction radii $R_{\mathrm{ex}}=(30 \mathrm{M}, 50 \mathrm{M}$, $75 M)$. For the oscillation amplitudes we get consistent results of $a_{1}=0.004 \pm 0.001, a_{2}=-0.004 \pm 0.001$, with statistical errors corresponding to the $95 \%$ confidence interval and rounded to one significant digit. We conclude that the asymptotic value of the dimensionless Kerr parameter is $a / M \approx 0.69$, which is consistent with the value 0.7 we obtained from the black hole horizon. Since the oscillation $a_{1} \cos \left(\alpha+\varphi_{1}\right)$, which has the periodicity of the kick velocity, is not consistent between the $m=-2,2$ modes, and the oscillation $a_{2} \cos \left(2 \alpha+\varphi_{2}\right)$ is of the same size, we conclude that both may be nonphysical, e.g. they could be due to gauge effects in the radiation extraction algorithm at finite radius (we suggest an alternative explanation in the next paragraph). It is plausible that such problems are more serious in the present case of large kicks than when the black hole system does not move with respect to the center of gravity. It would be interesting to analyze the present case more carefully e.g. along the lines discussed in [45].

Since the final spin of the black hole is close to the value for nonspinning black hole mergers, it appears that the individual, antialigned spins of the black holes do not contribute to the final angular momentum, but rather cancel approximately during merger. This is worth noting since based on the PN analysis and the numerical evolutions there is a small oscillating $z$ component of the spin of the individual black holes. At merger time, the $z$ component of 
the black hole spins is added to the spin of the merged black holes due to orbital motion. In principle it could happen that the initially small $S_{z}$ is enlarged greatly (as the PN calculation becomes inaccurate), e.g. it could happen that the separate spins precess significantly towards the $z$ axis and add significantly to the final angular momentum of the black hole. But this does not seem to happen, at best there is a small positive or negative contribution to the final spin depending on the momentary phase of the $S_{z}$ oscillation during merger, e.g. as we see in Fig. 13.

\section{EFFECTS OF RECOIL ON SNR AND TEMPLATE MATCH}

The gravitational recoil is essentially due to the symmetry breaking between the dominating modes $l=2, m=$ \pm 2 . A natural question is how this symmetry breaking is reflected in the overlap integrals of the gravitational waveforms. If the symmetry was not broken, the gravitational wave signal emitted towards the "north pole" would also provide the best template for the south pole. Similarly, for a sequence of waveforms that correspond to initial data that differ only in spin orientation (which we have parameterized by the angle $\alpha$ ), we can ask how much signal-to-noise ratio is lost when trying to detect the gravitational wave signal corresponding to some value of $\alpha$ with a template corresponding to a different value of $\alpha$.

Answering this question requires accurate waveforms, since any mismatch of waveforms can also be due to lack of numerical resolution, errors from the finite extraction radius (which can be significant, in particular, because the recoil velocity creates an asymmetry of the geometry of the extraction sphere), and the contribution of the initial junk radiation.

For the data we consider in this paper, the initial junk radiation cannot be separated from the main signal in a sufficiently clean fashion, neither can we obtain accurate error bars on the wave signals of the whole $\alpha$ series to really settle the above questions. Nevertheless, we are able to give some preliminary results.

We define the correlation function between two time series $x(t)$ and $y(t)$ for a time shift $\tau$ as

$$
R_{x y}(\tau)=\int_{-\infty}^{\infty} x(t) y^{\star}(t-\tau) d t,
$$

where a $\star$ denotes complex conjugation, and for simplicity we do not weight the integrand with the power spectral density corresponding to detector noise. Working with Fourier transforms

$$
x(t)=\int_{-\infty}^{\infty} \tilde{x}(f) e^{2 \pi i f t} d f,
$$

the correlation function can be written as

$$
R_{x y}(\tau)=\int_{-\infty}^{\infty} \tilde{x}(f) \tilde{y}^{\star}(f) e^{2 \pi i f \tau} d f
$$

in terms of Fourier transforms $\tilde{x}(f), \tilde{y}(f)$ of the time series. The function $R_{x y}(\tau)$ is thus simply the inverse Fourier transform of $\tilde{x}(f) \tilde{y}^{\star}(f)$. The value of $\tau$ for which $R_{x y}$ is maximal determines the time shift required to get the maximum correlation between $x(t)$ and $y(t)$. The selfcorrelation $R_{x x}$ is maximal for $\tau=0$ :

$$
R_{x x}(0)=\int_{-\infty}^{\infty}|\tilde{x}(f)|^{2} d f .
$$

More generally, the correlation function for $\tau=0$ yields the standard scalar product

$$
R_{x y}(0)=(x \mid y):=\int_{-\infty}^{\infty} \tilde{x}(f) \tilde{y}^{\star}(f) d f .
$$

The "match" that determines the efficiency of a template $y$ to identify a signal $x$ is defined as

$$
\mathcal{M}=\max _{\tau} \frac{\left|R_{x y}(\tau)\right|}{\sqrt{(x \mid x)(y \mid y)}},
$$

and correspondingly the mismatch can be defined as $1-$ $\mathcal{M}$. Note that in gravitational wave data analysis the orientation of a single detector actually reduces the signal to a real time series. We can now evaluate $\mathcal{M}$ for signals corresponding to different values of the initial spin angle $\alpha$ in our $\alpha$ series, or for signals corresponding to different angles in the sky for a given value of $\alpha$, say one with a large value of the recoil. From symmetry we expect that the match $\mathcal{M}$ when comparing the signals corresponding to the maximal difference in the kick within the $\alpha$ series $(\approx 5000 \mathrm{~km} / \mathrm{s}$ ) equals the match for the signals that correspond to the north and south poles for the maximal recoil case. Indeed we find a consistent match of $M \approx 0.94 \pm$ 0.01 both between the signals corresponding to the north and south poles for the $\alpha=0$ case (which is close to the maximal recoil), and for the maximal mismatch we find within the $\alpha$ series (which occurs roughly between the extreme cases of the recoil). Deviations of \pm 0.01 here come from comparing either the full complex waveform, or just $h_{+}$or $h_{\times}$. The uncertainties due to initial junk radiation, finite extraction radius, and numerical error may, however, be larger than $1 \%$. More accurate data than presented here will be required for conclusive error estimates. However, a detailed discussion of the dependence of the radiation signal on the angle $\alpha$ and the consequences for gravitational wave data analysis is beyond the scope of the present paper.

A related question is how much brighter the source appears in the direction opposite to the recoil, in which more radiation is emitted. We estimate the relative increase in SNR by computing the ratio of the norm of the strain $h$ for a given inclination angle $\theta$ to the strain measured at the south pole $(\theta=\pi)$ :

$$
\frac{\operatorname{SNR}(\theta)}{\operatorname{SNR}(\theta=\pi)}=\sqrt{\frac{(h(\theta) \mid h(\theta)}{(h(\theta=\pi) \mid h(\theta=\pi)}} .
$$




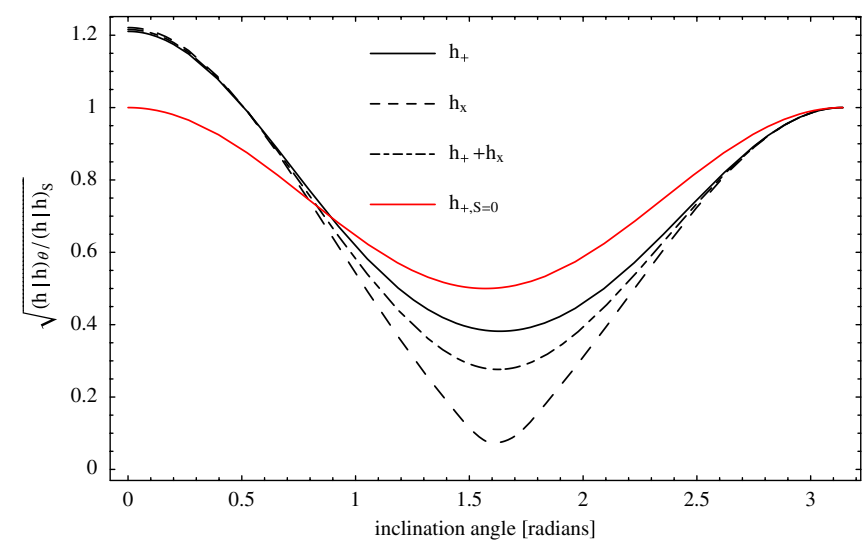

FIG. 14 (color online). The excess of signal compared to the south pole as defined in (13) is plotted as a function of the inclination angle $\theta$ for $h_{+}, h_{\times}$and $h_{+}-i h_{\times}$for the near extremal member of the $\alpha$ series $\alpha=0$. For comparison we also show the curve for the case without spin, when $h_{+}$is symmetric around $\theta=\pi / 2$. The excess of signal toward the north pole compared with the south pole is roughly $25 \%$.

In Fig. 14 we plot this ratio for the close-to-extreme case member of the $\alpha$ series, $\alpha=0$, for $h_{+}, h_{\times}$, and $h_{+}-i h_{\times}$. The excess of signal toward the north pole compared with the south pole is roughly $25 \%$. Note that this number may seem surprisingly small when compared to the excess of energy $E_{22} / E_{2-2} \approx 2.4$. However, there is no simple scaling relation between these two numbers: while at early times the signals in the north and south hemispheres show no significant deviation, there is a large deviation at late times. This implies a large deviation in the time derivatives of $h_{+,-}$, and it is the time derivative that determines the energy radiation; hence, the difference in energy radiated at the north and south poles is greater than the SNR difference.

\section{DISCUSSION}

We have discussed superkick configurations-two equal-mass black holes with spins antialigned and in the orbital plane-as a simple but extreme "test case" for phenomena associated with the large recoil velocities produced by spinning black hole binary systems. The high degree of symmetry results in the gravitational wave signal being dominated by the $l=2, m= \pm 2$ spherical harmonics, i.e., the recoil is with good accuracy proportional to the difference of energies radiated into the $l=2, m= \pm 2$ modes, as shown in Fig. 4.

The asymmetry here is rather strong and in the most extreme case $E_{22} / E_{2-2} \approx 2.4$. For gravitational wave detection the ratio of the amplitude of the strain $h$ is more relevant, and in the direction opposite to the recoil we find an excess of roughly $25 \%$ larger amplitude for the case with the maximum recoil.

For the large kicks one observes in the superkick configuration, one should certainly worry about the accuracy of the wave extraction. For the present paper we have been interested in a qualitative discussion rather than very high accuracy, but we point out that a procedure to improve the accuracy of wave extraction via the Newman-Penrose sca$\operatorname{lar} \Psi_{4}$ at finite radius has been discussed recently in [45]. An overall improvement in the accuracy of spinning black hole binary simulations should also be possible by employing higher-order spatial finite differencing [46] and using initial parameters based on PN inspiral calculations [47].

The main emphasis of this paper has been the comparison of the dynamics with post-Newtonian predictions. We have found that the $2.5 \mathrm{PN}$-accurate expressions given in [13] qualitatively describe the spin evolution and linear momentum radiation up to about $60 \mathrm{M}$ before merger. After that time the PN estimate of $d P_{z} / d t$ diverges from the numerical values. It is also after that time that we find the main contribution to the final kick of the merged black hole, and this explains why it is difficult to make accurate predictions of the kick by integrating the PN equations up to a cutoff separation. In order to accurately analytically model the recoil for superkick configurations (and possibly spinning black hole binary configurations in general) we suggest that a more sophisticated post-Newtonian treatment would be necessary, or a matching of PN methods during the early inspiral with a close-limit analysis of the merger and ringdown. It has recently been found [21] that a phenomenological formula for the final kick, based on the angular dependence of the terms in Eq. (B3), matches numerical data reasonably well. Having found that the precise form of (B3) fails to predict the linear momentum radiation in the regime when the majority of the linear momentum is radiated, it will be interesting to see how well such a phenomenological formula works for more general configurations, or if a more detailed analytic study will suggest a more generally applicable formula.

\section{ACKNOWLEDGMENTS}

We are grateful to P. Ajith, B. Krishnan, and A. Sintes for discussions regarding material in Sec. IV and to G. Schäfer for sharing insights on the post-Newtonian approach. This work was supported in part by DFG Grant No. SFB/Transregio 7 "Gravitational Wave Astronomy." We thank the DEISA Consortium (co-funded by the EU, FP6 Project No. 508830) for support within the DEISA Extreme Computing Initiative (www.deisa.org). Computations were performed at LRZ Munich and the Doppler and Kepler clusters at the Institute of Theoretical Physics of the University of Jena.

\section{APPENDIX A: INITIAL DATA PARAMETERS}

In the Bowen-York/puncture data that we use, we must choose parameters for the masses, separations, spins, and linear momenta of the two punctures. Most of the simulations studied in this paper are based on the MI configura- 
tion in [10], for which the momenta were chosen without any attempt to have the punctures move in quasicircular orbit (although the eccentricity in the resulting evolutions appears to be small). Often, however, one wishes to produce quasicircular orbits, and the momenta for our nonMI-based simulations are chosen to meet that requirement. In this appendix we describe our procedure for calculating those parameters. Note that in [47] we have described a procedure to further improve the "circularity" of the initial data by using parameters obtained from post-Newtonian inspirals.

In [24] we showed that a 3PN-accurate formula is sufficient to calculate initial momenta for nonspinning binaries with small initial separation. In the spinning case we make use of the results of Kidder [13]. They are in harmonic coordinates, but as we will see in Appendix D, the difference between harmonic coordinates, and the ADMTT gauge that we expect our evolutions to be in, are small. Kidder's Eq. (4.7) gives the orbital angular momentum of a binary in circular orbit as

$$
\begin{aligned}
\mathbf{L}= & \mu(M r)^{1 / 2} \hat{\mathbf{L}}_{N}\left\{1+2\left(\frac{M}{r}\right)-\frac{1}{4} \sum_{i=1,2}\left[\chi_{i}\left(\hat{\mathbf{L}}_{N} \cdot \hat{\mathbf{S}}_{i}\right)\right.\right. \\
& \left.\times\left(8 \frac{M_{i}^{2}}{M^{2}}+7 \eta\right)\right]\left(\frac{M}{r}\right)^{3 / 2}+\left[\frac{1}{2}(5-9 \eta)\right. \\
& \left.\left.-\frac{3}{4} \eta \chi_{1} \chi_{2}\left[\left(\hat{\mathbf{S}}_{1} \cdot \hat{\mathbf{S}}_{2}\right)-3\left(\hat{\mathbf{L}}_{N} \cdot \hat{\mathbf{S}}_{1}\right)\left(\hat{\mathbf{L}}_{N} \cdot \hat{\mathbf{S}}_{2}\right)\right]\right]\left(\frac{M}{r}\right)^{2}\right\} \\
& -\frac{1}{4} \mu(M r)^{1 / 2} \sum_{i=1,2}\left[\chi_{i} \hat{\mathbf{S}}_{i}\left(4 \frac{M_{i}^{2}}{M^{2}}+\eta\right)\right]\left(\frac{M}{r}\right)^{3 / 2}, \quad \text { (A1) }
\end{aligned}
$$

and the total angular momentum is $\mathbf{J}=\mathbf{L}+\mathbf{S}$. The variables in Eq. (A1) are as follows. The black holes are separated by a distance $r$ and have masses $M_{i}$, the total mass is $M=M_{1}+M_{2}$, and the mass ratio quantities are $\mu=M_{1} M_{2} / M$ and $\eta=\mu / M$. The black holes have spins $\mathbf{S}_{i}$, and $\chi_{i}=\left|S_{i}\right| / M_{i}^{2}$. The quantity $\hat{\mathbf{L}}_{N}$ denotes the unit vector in the direction of the angular momentum of a Newtonian system of nonspinning particles. It need not point in the same direction as the full orbital angular momentum $\mathbf{L}$ of the system, and we may exploit this freedom to uniquely find a momentum $\mathbf{P}$ that satisfies

$$
\mathbf{L}=\mu(\mathbf{r} \times \mathbf{P}) .
$$

The specific setup of our data is as follows. The punctures are placed on the $y$ axis and given momenta in the $x$ direction. The orbital angular momentum therefore has only one component and that points in the $z$ direction. In cases where the last term in Eq. (A1) has a component in the $x$ or $y$ directions, we tilt $\hat{\mathbf{L}}_{N}$ such that the last term is canceled out and $\mathbf{L}=L \hat{\mathbf{z}}$. Such a case will not arise in the situations considered in this paper; the last term in Eq. (A1) will always sum to zero and we can simply write $p_{x}=$ $\mp L / r$.

\section{APPENDIX B: POST-NEWTONIAN TREATMENT OF SPINNING BINARIES}

Consider two particles with masses $M_{1}$ and $M_{2}$, spins $\mathbf{S}_{1}$ and $\mathbf{S}_{2}$, located at positions $\mathbf{x}_{1}$ and $\mathbf{x}_{2}$. We define $\mathbf{x}=$ $\mathbf{x}_{1}-\mathbf{x}_{2}$ and $\mathbf{v}=d \mathbf{x} / d t$. Expressions for the evolution of the spins are given up to 2.5 post-Newtonian order in harmonic coordinates by Kidder [13],

$$
\begin{aligned}
\dot{\mathbf{S}}_{1}= & \frac{1}{r^{3}}\left\{\left(\mathbf{L}_{N} \times \mathbf{S}_{1}\right)\left(2+\frac{3}{2} \frac{M_{2}}{M_{1}}\right)-\mathbf{S}_{2} \times \mathbf{S}_{1}\right. \\
& \left.+3\left(\hat{\mathbf{n}} \cdot \mathbf{S}_{2}\right) \mathbf{n} \times \mathbf{S}_{1}\right\}, \\
\dot{\mathbf{S}}_{2}= & \frac{1}{r^{3}}\left\{\left(\mathbf{L}_{N} \times \mathbf{S}_{2}\right)\left(2+\frac{3}{2} \frac{M_{1}}{M_{2}}\right)-\mathbf{S}_{1} \times \mathbf{S}_{2}\right. \\
& \left.+3\left(\hat{\mathbf{n}} \cdot \mathbf{S}_{1}\right) \mathbf{n} \times \mathbf{S}_{2}\right\},
\end{aligned}
$$

where the Newtonian orbital angular momentum is given by $\mathbf{L}_{N}=\mu(\mathbf{x} \times \mathbf{v})$. Note that a particular spin supplementary condition has been chosen.

The radiated linear momentum is in turn given by Newtonian and spin-orbit contributions,

$$
\begin{aligned}
\dot{\mathbf{P}}_{N}= & -\frac{8}{105} \frac{\delta m}{M} \eta^{2}\left(\frac{M}{r}\right)^{4}\left\{\dot{r} \hat{\mathbf{n}}\left[55 v^{2}-45 \dot{r}^{2}+12 \frac{M}{r}\right]\right. \\
+ & \left.\mathbf{v}\left[38 \dot{r}^{2}-50 v^{2}-8 \frac{M}{r}\right]\right\}, \\
\dot{\mathbf{P}}_{\mathrm{SO}}= & -\frac{8}{15} \frac{\mu^{2} M}{r^{5}}\left\{4 \dot{r}(\mathbf{v} \times \boldsymbol{\Delta})-2 v^{2}(\hat{\mathbf{n}} \times \mathbf{\Delta})\right. \\
& -(\hat{\mathbf{n}} \times \mathbf{v})[3 \dot{r}(\hat{\mathbf{n}} \cdot \boldsymbol{\Delta})+2(\mathbf{v} \cdot \boldsymbol{\Delta})]\},
\end{aligned}
$$

where $\boldsymbol{\Delta}=M\left(\mathbf{S}_{2} / M_{2}-\mathbf{S}_{1} / M_{1}\right)$ and $\delta m=M_{1}-M_{2}$. Clearly the Newtonian contribution, which was used by Fitchett [48] to provide an early estimate of the recoil from the merger of nonspinning binaries, is zero in the equalmass case.

To evaluate Eqs. (B1) and (B3) one needs the particles' positions and velocities as a function of time, i.e., one needs to solve the post-Newtonian equations of motion. Alternatively, we can use the motions of the punctures calculated in our numerical simulations. This allows us to compare the precession of the spins and the radiated linear momentum with that predicted by post-Newtonian theory for the same motion. The results of this comparison are discussed in Sec. III E.

It is instructive to reduce Eqs. (B1)-(B3) to the special case of equal mass and $\pi$ symmetry. Equal masses $M_{1}=$ $M_{2}$ imply $\delta m=0$ and hence $\dot{\mathbf{P}}_{N}=0$. Furthermore, $\mu=$ $M / 4, \eta=1 / 4$, and $\boldsymbol{\Delta}=2\left(\mathbf{S}_{2}-\mathbf{S}_{1}\right)$.

For $\pi$ symmetry (which implies equal masses), the positions of the punctures $\mathbf{x}_{i}=\left(x_{i}, y_{i}, z_{i}\right)$ satisfy

$$
\left(x_{2}, y_{2}, z_{2}\right)=\left(-x_{1},-y_{1},+z_{1}\right)
$$

for all times. This implies for the relative position and 
velocity variables

$$
\mathbf{x}=\left(2 x_{1}, 2 y_{1}, 0\right), \quad \mathbf{v}=\left(2 v_{1 x}, 2 v_{1 y}, 0\right) .
$$

In other words, for $\pi$ symmetry the punctures can move in unison in the $z$ direction, and in general $z_{i}(t)$ will describe an accelerated motion. However, the PN Eqs. (B1)-(B3) are expressed in terms of $\mathbf{x}$ and $\mathbf{v}$, which describe the motion in coordinates in which the center of mass is at rest. Both $\mathbf{x}$ and $\mathbf{v}$ are orthogonal to the $z$ axis and lie in the $z=0$ plane. In particular, $\mathbf{v}$ does not have a component in the $z$ direction, so for general $z_{i}(t)$ the center-of-mass frame is an accelerated frame. A related statement is that in $\pi$ symmetry the orbital plane remains orthogonal to the $z$ axis, there is no orbital plane precession, and $\mathbf{L}_{N}=$ $\mu(\mathbf{x} \times \mathbf{v})$ is parallel to the $z$ axis at all times.

For $\pi$ symmetry the spins can be written as

$$
\mathbf{S}_{1}=\zeta+\sigma, \quad \mathbf{S}_{2}=\zeta-\sigma,
$$

where $\zeta$ and $\sigma$ are the components of the spin parallel and orthogonal to the $z$ axis, respectively. Hence the sum of the spins $\mathbf{S}=\mathbf{S}_{1}+\mathbf{S}_{2}$ points in the $z$ direction and the weighted spin difference $\Delta=2\left(\mathbf{S}_{2}-\mathbf{S}_{1}\right)$ is orthogonal to the $z$ axis,

$$
\mathbf{S}=2 \zeta, \quad \boldsymbol{\Delta}=-4 \sigma
$$

For the initial data we choose $\zeta=0$, but in general $\zeta$ is not constant in time. The time derivative of the spins can be written as

$$
\dot{\mathbf{S}}_{i}=\boldsymbol{\Omega}_{j} \times \mathbf{S}_{i}, \quad \boldsymbol{\Omega}_{j}=\frac{1}{r^{3}}\left(\frac{7}{2} \mathbf{L}_{N}-\mathbf{S}_{j}+3\left(\hat{\mathbf{n}} \cdot \mathbf{S}_{j}\right) \hat{\mathbf{n}}\right),
$$

where one part is precession about the $z$ axis due to the orbit-spin term, $\mathbf{L}_{N} \times \mathbf{S}_{i}$, but the axis of precession is in general not parallel to the $z$ axis due to the spin-spin terms. For $\pi$ symmetry we obtain

$$
\dot{\mathbf{S}}=-\frac{6}{r^{3}}(\hat{\mathbf{n}} \cdot \sigma)(\hat{\mathbf{n}} \times \sigma)=-\frac{3}{r^{3}}|\sigma|^{2} \sin (2 \alpha) \hat{\mathbf{z}},
$$

where $\hat{\mathbf{z}}$ is the unit vector in the $z$ direction and $\alpha$ is the angle between $\hat{\mathbf{n}}$ and $\sigma$. The $z$ component of the spins, $\zeta=$ $\mathbf{S} / 2$, oscillates with $\sin (2 \alpha(t))$. Since for negligible precession $\alpha(t)$ is equal to the orbital phase plus a phase shift, we expect two oscillations per orbit, which roughly agrees with observation, see Secs. III C and III E. For the weighted difference of the spins

$$
\begin{aligned}
\dot{\boldsymbol{\Delta}} & =-4 \dot{\sigma} \\
& =-\frac{7 M}{2 r^{2}}(\hat{\mathbf{n}} \times \mathbf{v}) \sigma-\frac{4}{r^{3}}(2 \sigma+3|\sigma| \cos (\alpha) \hat{\mathbf{n}}) \times \zeta,
\end{aligned}
$$

which contains precession due to $\mathbf{L}_{N}$ at order $1 / r^{2}$ and the spin-spin term at order $1 / r^{3}$. The term $\zeta \times \sigma$ describes a modulation of the precession about the $z$ axis since $\zeta$ oscillates around zero.

For the radiated linear momentum we note that for $\pi$ symmetry the three vectors $\hat{\mathbf{n}}, \mathbf{v}$, and $\boldsymbol{\Delta}$ in (B3) are orthogonal to the $z$ axis, and hence $\dot{\mathbf{P}}_{\text {SO }}$ is parallel to the $z$ axis as it should be. The angle between $\hat{\mathbf{n}}$ and $\mathbf{v}$ varies slowly over the entire inspiral from about $\pi / 2$ for circular orbits to a value less than $\pi$ for the plunge. The angle between the orbital vectors and $\boldsymbol{\Delta}$ oscillates with the orbital and precession time scales. Making the approximation that $\hat{\mathbf{n}} \cdot \mathbf{v} \approx 0$ and $\hat{\mathbf{n}} \times \mathbf{v} \approx v \hat{\mathbf{z}}$, we obtain

$$
\dot{\mathbf{P}}_{\mathrm{SO}} \approx-\frac{2}{15} \frac{M^{3}}{r^{5}}\left(7 \dot{r} v(\hat{\mathbf{n}} \cdot \sigma)+4 v^{2}\left(\frac{\mathbf{v}}{v} \cdot \sigma\right)\right) \hat{\mathbf{z}} .
$$

Even for quasicircular orbits where in addition we set $\dot{r}=$ 0 there will be radiation of linear momentum in the $z$ direction, which however averages to zero over time.

Note that in general there are two contributions, one proportional to $\dot{r} v$ and the other to $v^{2}$, and they are offset in phase depending on the angles between the spin and the orbital vectors. As the system approaches the plunge phase, the $\dot{r} v$ term should become as important as the $v^{2}$ effects. Note that we have not discussed the $\dot{\mathbf{P}}_{\mathrm{SS}}$ spin-spin contribution at next PN order, which could also be examined for potentially large contributions near the plunge, but in numerical simulations of head-on collisions the resulting kicks have been found to be small [49].

The PN expressions (B3) and the above discussion apply in the regime where the post-Newtonian approximations are valid. We see in Sec. IIIE that these expressions describe the radiation of linear momentum with reasonable accuracy up to about $50 M$ before merger.

\section{APPENDIX C: PN CALCULATION OF PUNCTURE MOTION}

In moving-puncture simulations we can readily track the motion of the punctures and record their positions $x(t)$ and velocities $v(t)=-\beta(t)$. We may then be tempted to make a Newtonian analogy and guess that the puncture's momentum is $P=M_{1} v$ for a black hole with mass $M_{1}$. However, when we compare this to the momentum specified in the initial data, the two values differ significantly. For example, evolve two equal-mass punctures with initial separation $D=8 M, P=0.14, M_{1}=M_{2}=0.5$. From the numerical puncture motion we find $M_{1} v \approx 0.075$; this value disagrees with $P$ by almost a factor of 2 .

During a simulation the "punctures" are at an infinite proper distance from their black holes' horizons [50,51], and we may worry that correctly physically interpreting the punctures' motions requires a thorough investigation of the gauge and geometry of the punctures as they evolve. In fact, the punctures' motions can be understood from a simple post-Newtonian analysis. 
Up to 2PN order, the Hamiltonian for two point particles in the ADMTT gauge and center-of-mass frame has been derived in [52,53].

From the Hamiltonian equations of motion,

$$
\dot{x}_{i}=\frac{\partial H}{\partial P_{i}}
$$

where $x_{i}$ is the separation vector between the two particles. At Newtonian order we recover $\dot{x}_{i}=P_{i} /(2 \mu)$. Up to $2 \mathrm{PN}$ order we have for circular orbits

$$
\begin{aligned}
\dot{x}_{i}= & \frac{P_{i}}{2 \mu}\left\{1-\frac{1}{c^{2}}\left(\frac{P^{2}(1-3 \eta)}{2 \mu^{2}}+\frac{M(3+\eta)}{R}\right)\right. \\
& +\frac{1}{c^{4}}\left(\frac{3 P^{4}\left(1-5 \eta+5 \eta^{2}\right)}{8 \mu^{4}}\right. \\
& \left.\left.-\frac{M P^{2}\left(-5+20 \eta+3 \eta^{2}\right)}{2 \mu^{2} R}+\frac{M^{2}(5+8 \eta)}{R^{2}}\right)\right\} .
\end{aligned}
$$

The more general expressions (removing the assumption of

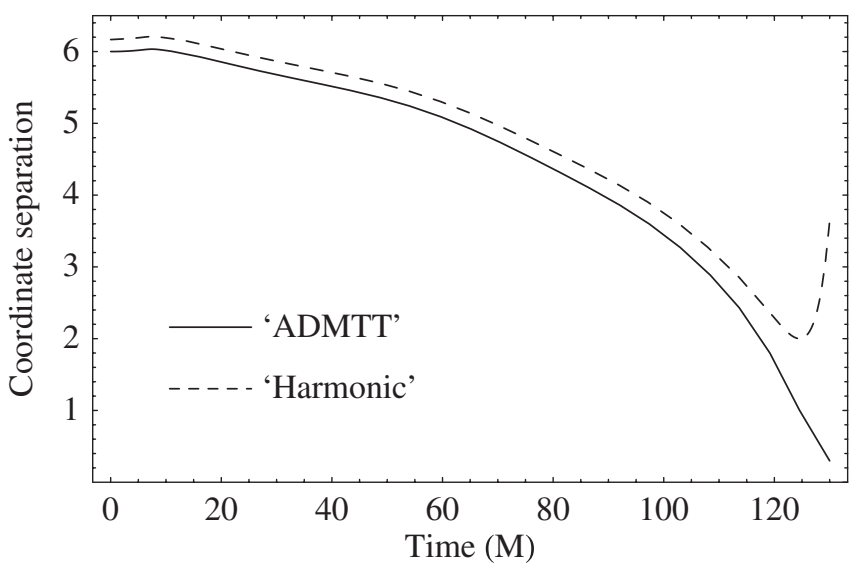

FIG. 15. Coordinate separation as a function of time for the D6 simulation, comparing the numerical data (presumed to be in ADMTT coordinates), and the same data transformed to harmonic coordinates. The difference is less than $10 \%$ up until about $15 \mathrm{M}$ before merger. It is also clear that after this time (when the "harmonic" curve turns upward) the PN approximations in the coordinate transformation break down.

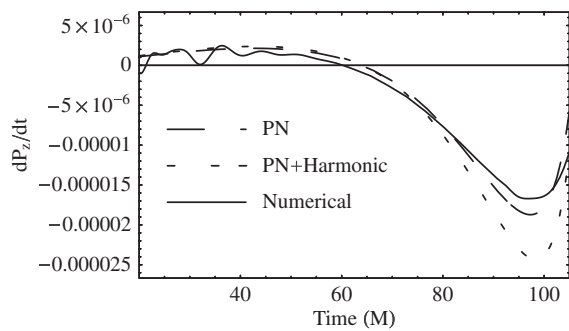

circular orbits), and the 3PN terms, will be omitted here for brevity. They can be readily calculated from the Hamiltonian in [53].

As an example, consider the $D=8 M$ quasicircular orbit parameters from the sequence presented in [54], for which $P=0.111 M$ and the orbital frequency is $M \Omega=0.0376$. From the orbital frequency we can calculate that the punctures will move at a speed $\dot{x}=0.150$, which is approximately equal to the observed value in a simulation. From the momentum, the Newtonian prediction of the speed is $\dot{x}_{N}=0.223$, which is far too high. The $1 \mathrm{PN}$ prediction is $\dot{x}_{1 \mathrm{PN}}=0.126$ (now the value is too small), and the $2 \mathrm{PN}$ and 3PN predictions are $\dot{x}_{2 \mathrm{PN}}=0.151$ and $\dot{x}_{3 \mathrm{PN}}=0.149$. The 2PN and 3PN predictions are both very close to the observed value.

In addition to providing a pleasing consistency between the dynamics observed in moving-puncture simulations and that predicted by post-Newtonian theory, this analysis is necessary when converting between ADMTT and harmonic gauges in Appendix D, where we will need to invert equations like (D1) to estimate the black holes' momenta as a function of time from the puncture motion.

\section{APPENDIX D: ADMTT TO HARMONIC TRANSFORMATION}

Our initial data are, up to $2 \mathrm{PN}$ order, in the ADMTT gauge [35]. The post-Newtonian expressions for spin evolution and linear momentum radiation listed in Appendix B are in the harmonic gauge. Although it is not clear how closely our evolved data adhere to the ADMTT gauge, it is nonetheless useful to assume that they remain in the ADMTT gauge and transform the results to the harmonic gauge and see how different they are.

A transformation between ADMTT and harmonic coordinates is provided up to $2 \mathrm{PN}$ order by Damour and Schäfer [52]. If $\mathbf{x}_{i}$ are the ADMTT coordinates of the $i$ th particle and $\mathbf{X}_{i}$ are the corresponding harmonic coordinates, then the transformation for a binary system is

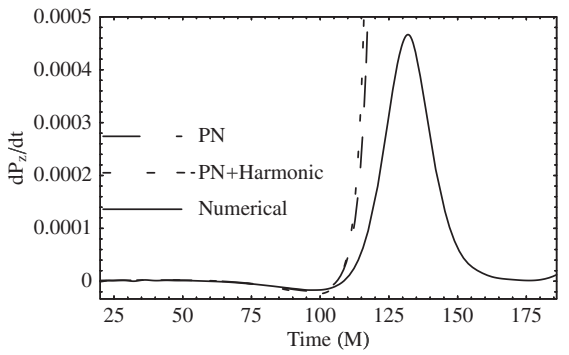

FIG. 16. Comparison of the numerical $d P_{z} / d t$ with that predicted by Eq. (B3), using both the numerical puncture positions and momenta in ADMTT coordinates, and those transformed to harmonic coordinates. At early times there is good qualitative agreement between all three approaches. At late times (right-hand panel) the PN and numerical values diverge, as discussed in Sec. IIIE. 


$$
\begin{aligned}
\mathbf{X}_{i}= & \mathbf{x}_{i}+M_{i}\left\{\mathbf{n}\left(\frac{5}{8} v_{j}^{2}-\frac{1}{8}\left(\mathbf{n} \cdot \mathbf{v}_{j}\right)^{2}+\frac{7 M_{i}}{4 R}+\frac{M_{j}}{4 R}\right)\right. \\
& \left.+\left(\frac{1}{2} \mathbf{v}_{i}-\frac{7}{4} \mathbf{v}_{j}\right)\left(\mathbf{n} \cdot \mathbf{v}_{j}\right)\right\} .
\end{aligned}
$$

The velocities $v_{\{a, b\}}$ in Eq. (D1) are not the coordinate speeds but are instead $v_{i}=p_{i} / m_{i}$, and the momenta $p_{i}$ must be determined by the procedure described in Appendix C.

When the particles are far apart and moving slowly, the coordinates $\mathbf{x}_{i}$ and $\mathbf{X}_{i}$ will not differ much. In Fig. 15, which shows results from the D6 simulation, we show the separation between the punctures in the numerical coordinates as a function of time, and in the coordinates after the transformation (D1). The coordinates differ by less than $10 \%$ up until about $15 \mathrm{M}$ before merger. After that time we do not expect the coordinate transformation (which is accurate up to only $2 \mathrm{PN}$ order) to be reliable. However, for most of the evolution we see that the differences between the two coordinate choices are not dramatic. A comparison of numerical and PN calculations of $d P_{z} / d t$ (as described in Sec. III E) in Fig. 16 also shows that the results are similar at early times, before the PN result diverges. Note that when the puncture motion in ADMTT coordinates is used in the (harmonic) PN formula (B3), the curve is closer to the numerical result than when we use the puncture motion in harmonic coordinates. However, since this agreement occurs just before the time when the PN and numerical values seriously diverge, we do not take this agreement too seriously.

The main conclusion of this analysis is that the difference in results between using ADMTT and harmonic dynamical quantities in PN expressions is less than the uncertainty inherent in the PN expressions themselves. We therefore continue to use the raw numerical data, in ADMTT coordinates, for most of the analysis presented in this paper.
[1] S. G. Hahn and R. W. Lindquist, Ann. Phys. (N.Y.) 29, 304 (1964).

[2] F. Pretorius, Phys. Rev. Lett. 95, 121101 (2005).

[3] M. Campanelli, C. O. Lousto, P. Marronetti, and Y. Zlochower, Phys. Rev. Lett. 96, 111101 (2006).

[4] J. G. Baker, J. Centrella, D.-I. Choi, M. Koppitz, and J. van Meter, Phys. Rev. Lett. 96, 111102 (2006).

[5] W. B. Bonnor and M. A. Rotenberg, Proc. R. Soc. A 265 , 109 (1961).

[6] A. Peres, Phys. Rev. 128, 2471 (1962).

[7] J. D. Bekenstein, Astrophys. J. 183, 657 (1973).

[8] A. Loeb, Phys. Rev. Lett. 99, 041103 (2007).

[9] E.W. Bonning, G. A. Shields, and S. Salviander, arXiv:0705.4263.

[10] J.A. González, M.D. Hannam, U. Sperhake, B. Brügmann, and S. Husa, Phys. Rev. Lett. 98, 231101 (2007).

[11] M. Campanelli, C. O. Lousto, Y. Zlochower, and D. Merritt, Phys. Rev. Lett. 98, 231102 (2007).

[12] M. Campanelli, C. O. Lousto, Y. Zlochower, and D. Merritt, Astrophys. J. 659, L5 (2007).

[13] L. E. Kidder, Phys. Rev. D 52, 821 (1995).

[14] M. Campanelli, C. O. Lousto, Y. Zlochower, and D. Merritt (2007), version 1, http://www.arxiv.org/abs/gr-qc/ $0701164 \mathrm{v} 1$.

[15] J. G. Baker, J. Centrella, D.-I. Choi, M. Koppitz, J. van Meter, and M. C. Miller, Astrophys. J. 653, L93 (2006).

[16] F. Herrmann, I. Hinder, D. Shoemaker, and P. Laguna, Classical Quantum Gravity 24, S33, (2007).

[17] F. Herrmann, I. Hinder, D. Shoemaker, P. Laguna, and R. A. Matzner, arXiv:gr-qc/0701143.

[18] M. Koppitz, D. Pollney, C. Reisswig, L. Rezzolla, J. Thornburg, P. Diener, and E. Schnetter, Phys. Rev. Lett. 99, 041102 (2007).
[19] M. Campanelli, C. O. Lousto, Y. Zlochower, and D. Merritt, Astrophys. J. 659, L5 (2007).

[20] W. Tichy and P. Marronetti, Phys. Rev. D 76, 061502 (2007).

[21] F. Herrmann, I. Hinder, D. M. Shoemaker, P. Laguna, and R. A. Matzner, Phys. Rev. D 76, 084032 (2007).

[22] J. A. González, U. Sperhake, B. Brügmann, M. Hannam, and S. Husa, Phys. Rev. Lett. 98, 091101 (2007).

[23] J.D. Schnittman and A. Buonanno, arXiv:astro-ph/ 0702641.

[24] B. Brügmann, J. A. González, M. Hannam, S. Husa, U. Sperhake, and W. Tichy, Phys. Rev. D 77, 024027 (2008).

[25] B. Brügmann, W. Tichy, and N. Jansen, Phys. Rev. Lett. 92, 211101 (2004).

[26] U. Sperhake, Phys. Rev. D 76, 104015 (2007).

[27] J. M. Bowen and J. W. York, Phys. Rev. D 21, 2047 (1980).

[28] S. Brandt and B. Brügmann, Phys. Rev. Lett. 78, 3606 (1997).

[29] M. Ansorg, B. Brügmann, and W. Tichy, Phys. Rev. D 70, 064011 (2004).

[30] M. Shibata and T. Nakamura, Phys. Rev. D 52, 5428 (1995).

[31] T.W. Baumgarte and S. L. Shapiro, Phys. Rev. D 59, 024007 (1998).

[32] J. W. York, in Sources of Gravitational Radiation, edited by L. L. Smarr (Cambridge University Press, Cambridge, England, 1979), pp. 83-126.

[33] M. Campanelli, C. O. Lousto, Y. Zlochower, B. Krishnan, and D. Merritt, Phys. Rev. D 75, 064030 (2007).

[34] D. R. Fiske, J. G. Baker, J. R. van Meter, D. Choi, and J. M. Centrella, Phys. Rev. D 71, 104036 (2005).

[35] P. Jaranowski and G. Schäfer, Phys. Rev. D 57, 7274 (1998). 
[36] M. Hannam, S. Husa, U. Sperhake, B. Brügmann, and J. A. González, Phys. Rev. D 77, 044020 (2008).

[37] M. Favata, S. A. Hughes, and D. E. Holz, Astrophys. J. 607, L5 (2004).

[38] C. F. Sopuerta, N. Yunes, and P. Laguna, Phys. Rev. D 74, 124010 (2006).

[39] C. F. Sopuerta, N. Yunes, and P. Laguna, Astrophys. J. 656, L9 (2007).

[40] L. Blanchet, M. S. S. Qusailah, and C. M. Will, Astrophys. J. 635, 508 (2005)

[41] T. Damour and A. Gopakumar, Phys. Rev. D 73, 124006 (2006).

[42] S. Detweiler, Astrophys. J. 239, 292 (1980).

[43] E. W. Leaver, Proc. R. Soc. A 402, 285 (1985).

[44] E. Berti, V. Cardoso, and C. M. Will, Phys. Rev. D 73, 064030 (2006).

[45] L. Lehner and O. M. Moreschi, Phys. Rev. D 76, 124040 (2007).

[46] S. Husa, J. A. González, M. Hannam, B. Brügmann, and U. Sperhake, arXiv:0706.0740.
[47] S. Husa, M. Hannam, J. A. González, U. Sperhake, and B. Brügmann, Phys. Rev. D 77, 044037 (2008).

[48] M. J. Fitchett, Mon. Not. R. Astron. Soc. 203, 1049 (1983).

[49] D.-I. Choi, B. J. Kelly, W.D. Boggs, J. G. Baker, J. Centrella, and J. van Meter, Phys. Rev. D 76, 104026 (2007).

[50] M. Hannam, S. Husa, D. Pollney, B. Brügmann, and N. Ó Murchadha, Phys. Rev. Lett. 99, 241102 (2007).

[51] M. Hannam, S. Husa, N. Ó Murchadha, B. Brügmann, J. A. González, and U. Sperhake, J. Phys. Conf. Ser. 66, 012047 (2007).

[52] T. Damour and G. Schäfer, Gen. Relativ. Gravit. 17, 879 (1985).

[53] T. Damour and G. Schäfer, Nuovo Cimento Soc. Ital. Fis. A 10, 123 (1988).

[54] W. Tichy and B. Brügmann, Phys. Rev. D 69, 024006 (2004). 